\title{
BMJ Open Facilitators and barriers to the effective implementation of the individual maternal near-miss case reviews in low/ middle-income countries: a systematic review of qualitative studies
}

\author{
Marzia Lazzerini, ${ }^{1}$ Margherita Ciuch, ${ }^{1}$ Silvia Rusconi, ${ }^{2}$ Benedetta Covi ${ }^{1}$
}

To cite: Lazzerini M, Ciuch M, Rusconi S, et al. Facilitators and barriers to the effective implementation of the individual maternal near-miss case reviews in low/middle-income countries: a systematic review of qualitative studies. BMJ Open 2018;8:e021281. doi:10.1136/ bmjopen-2017-021281

- Prepublication history and additional material for this paper are available online. To view these files, please visit the journal online (http://dx.doi. org/10.1136/bmjopen-2017021281).

Received 21 December 2017 Revised 19 April 2018 Accepted 20 April 2018
Check for updates

${ }^{1}$ WHO Collaborating Centre for Maternal and Child Health, Institute for Maternal and Child Health IRCCS Burlo Garofolo,

Trieste, Italy

${ }^{2}$ Department of Obstetrics and Gynecology, Hospital of Padova, Padova, Italy

Correspondence to

Dr Marzia Lazzerini;

marzia.lazzerini@burlo.trieste.it

\section{ABSTRACT}

Background The maternal near-miss cases review (NMCR), a type of clinical audit, proved to be effective in improving quality of care and decreasing maternal mortality in low/middle-income countries (LMICs). However, challenges in its implementation have been described.

Objectives Synthesising the evidence on facilitators and barriers to the effective implementation of NMCR in LMICs.

Design Systematic review of qualitative studies. Data sources MEDLINE, LILACS, Global Health Library, SCI-EXPANDED, SSCI, Cochrane library and Embase were searched in December 2017.

Eligibility criteria for selecting studies Qualitative studies exploring facilitators and/or barriers of implementing NMCR in LMIC were included.

Data extraction and synthesis Two independent reviewers extracted data, performed thematic analysis and assessed risk of bias.

Results Out of 25361 papers retrieved, 9 studies from Benin, Brazil, Burkina Faso, Cote D'Ivoire, Ghana, Malawi, Morocco, Tanzania, Uganda could be included in the review. The most frequently reported barriers to NMCR implementation were the following: absence of national guidelines and local protocols; insufficient training on how to perform the audit; lack of leadership, coordination, monitoring and supervision; lack of resources and work overload; fear of blame and punishment; poor knowledge of evidenced-based medicine; hierarchical differences among staff and poor understating of the benefits of the NMCR. Major facilitators to NMCR implementation included: good leadership and coordination; training of all key staff; a good cultural environment; clear staff's perception on the benefits of conducting audit; patient empowerment and the availability of external support.

Conclusions In planning the NMCR implementation in LMICs, policy-makers should consider actions to prevent and mitigate common challenges to successful NMCR implementation. Future studies should aim at documenting facilitators and barriers to NMCR outside the African Region.
Strengths and limitations of this study

- This review fills a gap in evidence synthesis by systematically reporting scientific literature on facilitators and barriers to effective implementation of near-miss cases review (NMCR) in low/middle-income countries (LMICs).

- Findings of this review are limited by the paucity of existing scientific reports: although the NMCR approach has been used in many countries (such as in Europe, Central Asia, South East Asia, Latin America and the Caribbean), there has been relatively few formal studies exploring facilitators and barriers to effective NMCR implementation.

- Despite the above-described limitation, this review retrieved an appreciable number of good-quality studies from the African Region and provides a list of recommendations relevant for both researchers and policy-makers for facilitating effective NMCR implementation in LMICs.

\section{BACKGROUND}

Ensuring adequate quality of healthcare is a primary objective of the WHO Global Strategy for Women's, Children's and Adolescent's Health 2016-2030. ${ }^{1}$ Quality in healthcare is recognised as essential for the health and well-being of the population and as a basic aspect of human rights. ${ }^{2} 3$

Among different approaches aiming at improving quality of care in maternity services, the maternal near-miss cases review (NMCR) approach was promoted by WHO and partners since 2004 within the strategy Beyond the Numbers. ${ }^{4}$ A maternal near-miss case is defined as a woman who nearly died but survived a complication that occurred during pregnancy, childbirth or within 6 weeks after pregnancy. ${ }^{5}$ The facility-based individual NMCR cycle is defined as a type of criterion-based audit seeking to improve maternal and perinatal healthcare and 
outcomes by conducting a review, at hospital level, of the care provided to maternal near-miss cases. ${ }^{5}$ Based on the findings of the case review, actions for improving quality of care are proposed and agreed by hospital staff. ${ }^{5}$ Beside reviewing clinical management, the NMCR can cover other domains involved with care delivery, including availability of essential equipment, staffing, training, policies and organisation of services. ${ }^{5}$ The bottom-up approach of the NMCR aims at ensuring local ownership and at facilitating team-building dynamics. ${ }^{5}$

The NMCR have been promoted in the last 20 years as a way to audit case management more acceptable for health workers than mortality audits. ${ }^{4-6}$ In most facilities, the number of maternal deaths is usually insufficient or not representative enough to allow reliable policy guidance. ${ }^{4}$ Near-miss cases occur more frequently than maternal deaths and their review can inform on both strengths and weaknesses in the process of care. Moreover, discussing cases of deaths may have legal implication and may be perceived as challenging by hospital staff, ${ }^{4}$ while the review of near-miss cases has showed an overall higher acceptability. ${ }^{4-6}$

A systematic review highlighted that the implementation of the NMCR cycle may significantly decrease maternal mortality (OR 0.77 , 95\% CI 0.61 to 0.98) in high burden countries and can improve quality of care when measured against predefined standards. ${ }^{7}$ However, a number of challenges hampering successful implementation of the NMCR were also reported. ${ }^{7}$ Knowledge on factors affecting the successful NMCR implementation can help policy-makers and development partners in better planning the intervention. Given the lack of other reviews exploring this question, the objective of this paper was to systematically synthesise the evidence on facilitators and barriers to effective NMCR implementation in low/middle-income countries (LMICs).

\section{METHODS}

\section{Search strategy and eligibility criteria}

In conducting this review, we followed the guidelines reported in the PRISMA (Preferred Reporting Items for Systematic Reviews and Meta-Analyses) ${ }^{8}$ and ENTREQ statement to enhance transparency in reporting of qualitative evidence synthesis ${ }^{9}$ (see online supplementary appendices 1 and 2). A protocol including detailed methods of the review was developed before starting the review.

We searched up to December 2017 the following databases, with no language restrictions: MEDLINE through PubMed (from 1956); LILACS through the Virtual Health Library (no date restrictions); Global Health Library (WHO website, no date restrictions); Science Citation Index Expanded (SCI-EXPANDED) and Social Sciences Citation Index (SSCI) through Web of Science (no date restrictions); Cochrane library (no date restrictions) and Embase through OVID (from 1996). The search strategy

\section{Box 1 Search strategy}

PubMed, Date: 1 December 2017, Total retrieved: 5661

"near miss" OR (audit AND (obstetric* OR matern* OR pregnan* OR woman OR women))

Lilacs, Date: 1 December 2017, Total retrieved: 231

(TW:near miss OR MH:near miss) OR ((TW:audit OR MH:audit OR

TW:auditoria OR MH:auditoria OR auditoría) AND (gravid\$ OR pregnan\$

OR enceint\$ OR embarazad\$ OR obstetr\$ OR mulher\$ OR mujer\$ OR femme\$ OR woman OR women OR matern\$))

Global Idex Medicus Date: 1 December 2017, Total retrieved: 7876 (TW:near miss OR MH:near miss) OR ((TW:audit OR MH:audit OR TW:auditoria OR MH:auditoria OR auditoría) AND (gravid\$ OR pregnan\$ OR enceint\$ OR embarazad\$ OR obstetr\$ OR mulher\$ OR mujer\$ OR femme\$ OR woman OR women OR matern\$))

Web of Science Date: 1 December 2017, Total retrieved: 5322

$\mathrm{TS}=$ "near miss" OR (TS=audit AND TS=(gravid* OR pregnan* OR obstetr* OR woman OR women OR matern*))

Cochrane Library Date: 1 December 2017, Total retrieved: 344

"near miss" OR (audit AND (gravid* or pregnan* or obstetr* or woman or women or matern*))

\section{EMBASE Date: 1 December 2017, Total retrieved: 5927}

1. ("near miss" or audit).ab. (34259)

2. (obstetric* or matern* or pregnan* or woman or women).ab. (1057153)

3. 1 and 2 (4764)

4. ("near miss" or audit).ti. (13725)

5. (obstetric* or matern* or pregnan* or woman or women).ti. (325314)

6. 4 and 5 (724)

7. 3 or $6(4962)$

is reported in box 1. Manual searches of reference lists were also performed.

Studies were eligible for inclusion if they explored facilitators and/or barriers of implementing the NMCR, either by collecting personal views of hospital staff or of patients, in an LMIC (defined as for the World Bank definition ${ }^{10}$ at the time when the study was conducted). Both studies using the most recent WHO definition of a maternal near-miss case $^{11}$ developed in year 2011, or locally adapted definitions (such as locally developed disease-specific definitions) were considered for inclusion. Studies reporting facilitators and barriers to effective NMCR implementation merely as the author's opinion (eg, in the section Discussion) and not as a result of a dedicated analysis were excluded. Abstracts and unpublished technical reports were also not eligible for inclusion. Studies on newborn near-miss cases were not included.

\section{Data collection and analysis}

Studies were selected for inclusion by two independent researchers. The full text of all eligible citations was examined in detail. Two researchers extracted data from included studies, using a prepiloted data extraction form. Any disagreement was solved via discussion between the two researchers and consensus sought through a third researcher. 
Two authors independently extracted information regarding the study setting, the study sample, methods and tools used for data collection and data analysis. Two authors independently created a spreadsheet with all facilitators and barriers reported in included studies and used thematic analysis methods to conduct initial open coding on each relevant text unit. In the initial round of coding, main emerging themes were synthesised and these were intentionally very broad in order to capture the overarching core themes. As a second step, each theme was further analysed to develop the axial coding scheme. Axial coding is widely accepted in qualitative literature as a sufficient method to disaggregate core themes during qualitative analysis. ${ }^{12-14}$ Two researchers independently applied the axial codes systematically to the data by handsorting the text units into themes and subthemes. Any disagreement on thematic analysis was solved by discussion between the two authors and consensus sought through a third author. Final results are reported in a table, providing the first-order, second-order and thirdorder themes. Excel and Word were used as software of data extraction.

The quality of studies was evaluated by two authors independently using the Critical Appraisal Skills Programme (CASP) assessment tool for qualitative studies. ${ }^{15}$

Three authors inferred barriers and facilitators reported in the included studies and captured by the descriptive themes, and developed key recommendations for effective NMCR implementation, in line with methods used by previous reviews. ${ }^{14}$ This process was performed first independently by each author and then as a group until consensus was reached.

\section{Patient and public involvement}

Patients were not directly involved in this study. However, the development of the research question and outcome measures was informed by patient experience, as previously reported in literature. ${ }^{2-5}$ For example, in revising studies, we evaluated whether patient views were considered, and the general attitude of service providers towards patients.

\section{RESULTS}

\section{Characteristics of the studies}

The systematic search yielded a total of 25361 records (figure 1). Overall, nine studies ${ }^{16-24}$ met the inclusion criteria (table 1). Of these, seven studies were held in countries in the African Region: Benin, ${ }^{21}{ }^{24}$ Burkina Faso, ${ }^{24}$ Cote D'Ivore,${ }^{24}$ Ghana, ${ }^{24}$ Malawi, ${ }^{20}$ Morocco,${ }^{22} 24$ Tanzania ${ }^{19}$ and Uganda. ${ }^{16}$ Two reports contributed on one study from Brazil. ${ }^{17} 18$

Most studies were conducted in low-income countries, with the exception of the studies in Morocco and Brazil (middle-income countries). Three studies were conducted in an urban setting, ${ }^{16} 2324$ one in a rural

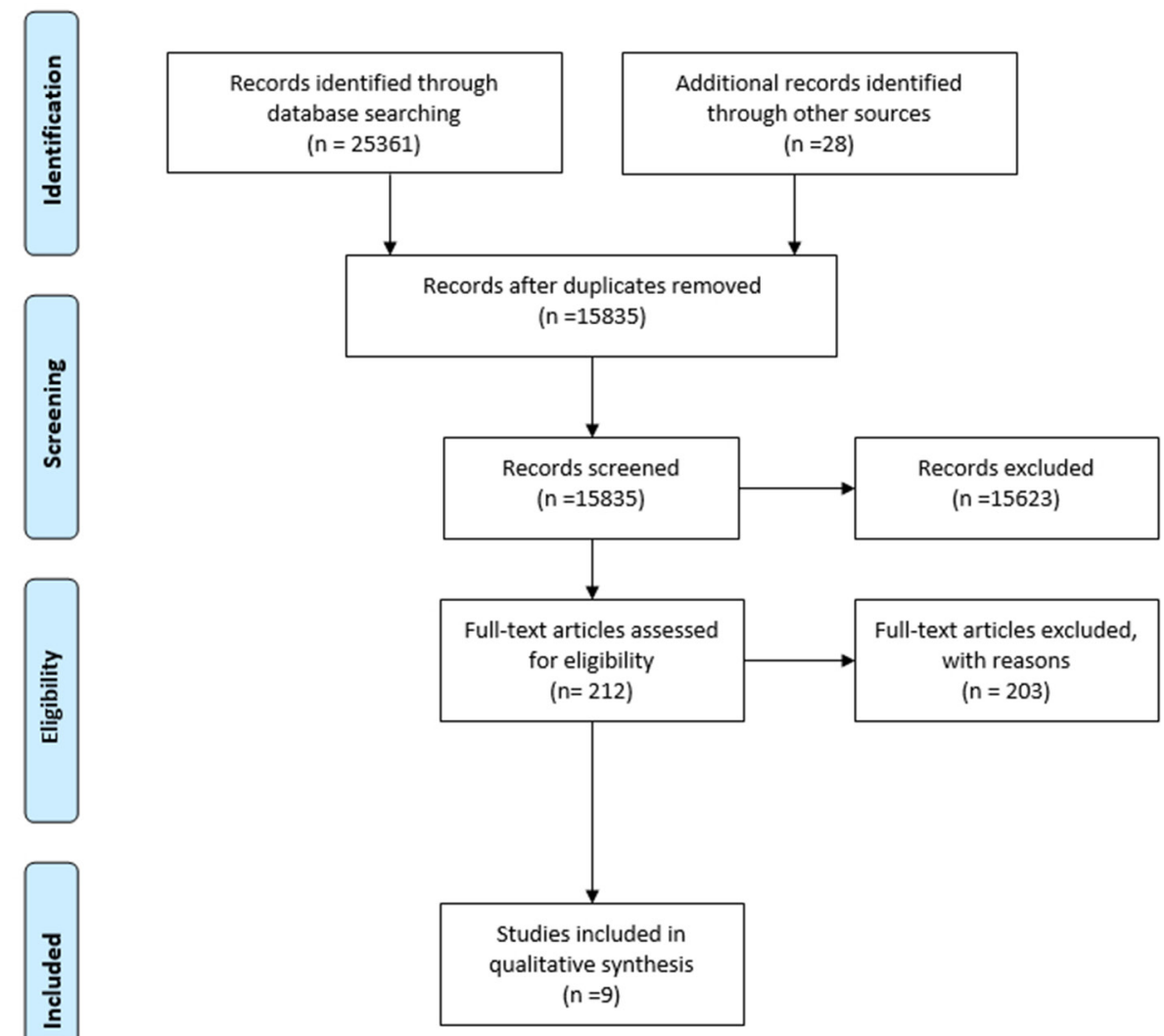

Figure 1 Study flow diagram. 
Table 1 Study context and population

\begin{tabular}{|c|c|c|c|c|c|c|c|}
\hline Study & Country & $\begin{array}{l}\text { World Bank } \\
\text { classification* }\end{array}$ & Setting & $\begin{array}{l}\text { Hospital } \\
\text { (n) }\end{array}$ & Hospital type $^{*}$ & $\begin{array}{l}\text { Sample } \\
\text { staff (n) }\end{array}$ & Staff type* \\
\hline Kayiga et al, $2016^{16}$ & Uganda & $\mathrm{L}$ & Urban & 1 & Tertiary hospital & 40 & $D, I, R$ \\
\hline $\begin{array}{l}\text { Gomez Luz et al, } 2014^{17} \\
\text { Gomez Luz et al, } 2014^{18}\end{array}$ & Brazil & UM & Mixed & 27 & $\begin{array}{l}\text { Mixed (all teaching } \\
\text { hospitals but } 5 \text { secondary } \\
\text { level, } 22 \text { tertiary level) }\end{array}$ & 122 & C, PI, MA \\
\hline Hamersveld et al, $2012^{19}$ & Tanzania & $\mathrm{L}$ & Rural & 1 & District hospital & 23 & $\mathrm{D}, \mathrm{C}, \mathrm{M}, \mathrm{N}, \mathrm{MA}$ \\
\hline Hutchinson et al, $2010^{21}$ & Benin & $\mathrm{L}$ & Mixed & 5 & $\begin{array}{l}\text { Mixed (two national } \\
\text { university hospitals, one } \\
\text { regional, one district, one } \\
\text { missionary }\end{array}$ & 10 & MA, HW \\
\hline Muffler et al, $2007^{22}$ & Morocco & LM & Mixed & 13 & Mixed & 56 & $\begin{array}{l}\text { MA, M, N, D, I, } \\
C, R\end{array}$ \\
\hline
\end{tabular}

${ }^{*} \mathrm{~L}$, low income; LM, lower middle income; UM, uppermiddle income (countries are classified based on the years when the study was performed).

C, coordinator, D, doctors, I, in charge; HW, health workers; I, investigators; M, midwives; MA, manager; n, nurses;

$\mathrm{PI}$, principal investigator; R,resident.

area, ${ }^{19}$ four in a mixed setting ${ }^{1720-22}$ and one not clarified this information. Overall, there were four large-tomiddle-sized studies including a conspicuous number of hospitals: 27 maternities in the Brazilian study ${ }^{17}{ }^{18} ; 13$ facilities in a study in Morocco ${ }^{22}$; 12 hospitals in a multicountry study ${ }^{24}$ and 5 in a study from Benin. ${ }^{21}$ One study in Malawi included two hospitals, ${ }^{20}$ while the remaining three studies included one single facility. ${ }^{16} 1923$ Number of staff interviewed (and/or included in the focus group) varied from a maximum of 162 people $^{24}$ to a minimum of $10 .{ }^{21}$ All studies collected the views of hospital staff, while none reported the views of patients.

In terms of methodology (table 2), most studies were conducted 1-2years after the start of the NMCR implementation, with only two studies ${ }^{21} 22$ being performed several years after. All studies used interviews as the main tool for data collection. In addition, two evaluations used focus group discussion, ${ }^{16}{ }^{20}$ three used direct observation of the NMCR session ${ }^{192024}$ and two evaluated notes from the NMCR sessions and other related documents. ${ }^{23} 24$ Five studies explicitly stated that the investigation was conducted by a researcher who was external from the study context, ${ }^{1720212324}$ while the others did not fully clarify the relationship between the interviewer and the participants. Other methods related to data collection and analyses are reported in table 2.

Quality of the studies according to the CASP criteria is reported in table 3 . Three studies matched all criteria for quality and were rated as 'high quality', ${ }^{17} 2123$ while the remaining studies were rated as of moderate quality. ${ }^{1619202224}$

\section{Barriers and facilitators}

Table 4 synthesises the first-order, second-order and third-order themes identified. Factors were divided into national-level factors, facility-level factors and external partners factors.

National level factors

\section{National standards}

Absence of national case management protocols ${ }^{16}$ was reported as a barrier to the effective implementation of NMCR.

\section{Leadership and coordination mechanisms}

Facilitators of effective NMCR implementation described by health workers included general commitment of health authorities ${ }^{20}$ and the establishment of effective coordination mechanisms, such as effective task allocation, ${ }^{17}$ networking support among facilities, ${ }^{24}$ availability of a standard form for reporting, ${ }^{21}$ effective monitoring and quality assessment. ${ }^{17} 21$ Commitment to training ${ }^{20}$ and integration of audits into medical and midwifery school curricula ${ }^{21}$ were also reported as facilitators.

Barriers to effective NMCR implementation included absence of directives from health authority ${ }^{22}$ and pressure from competing programme activities or interests. ${ }^{21} 22$

Facility level factors

National guidelines and standards

Absence of case management protocols ${ }^{16}$ at facility level was reported as key barrier in implementing the NMCR. ${ }^{16}$ 


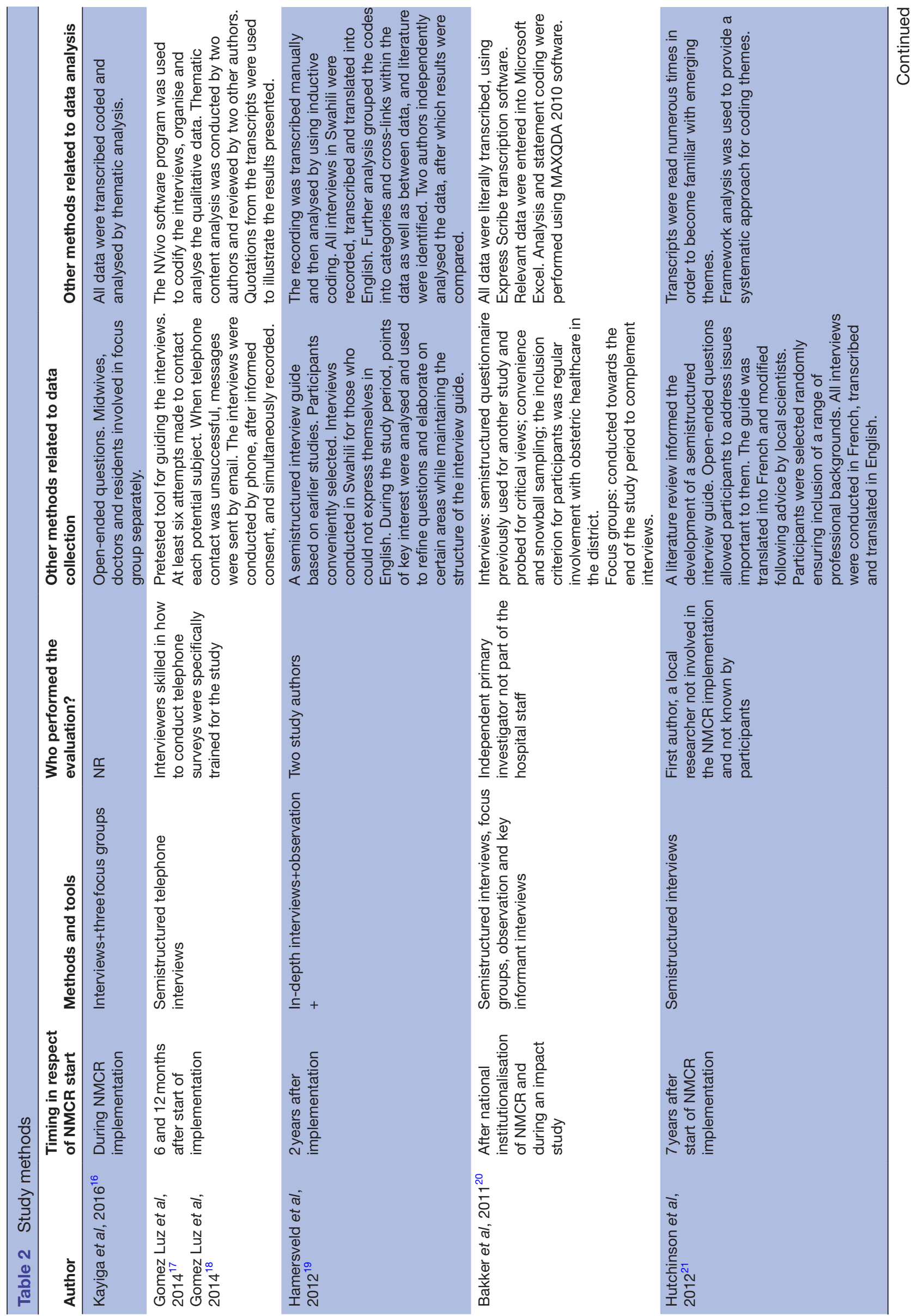




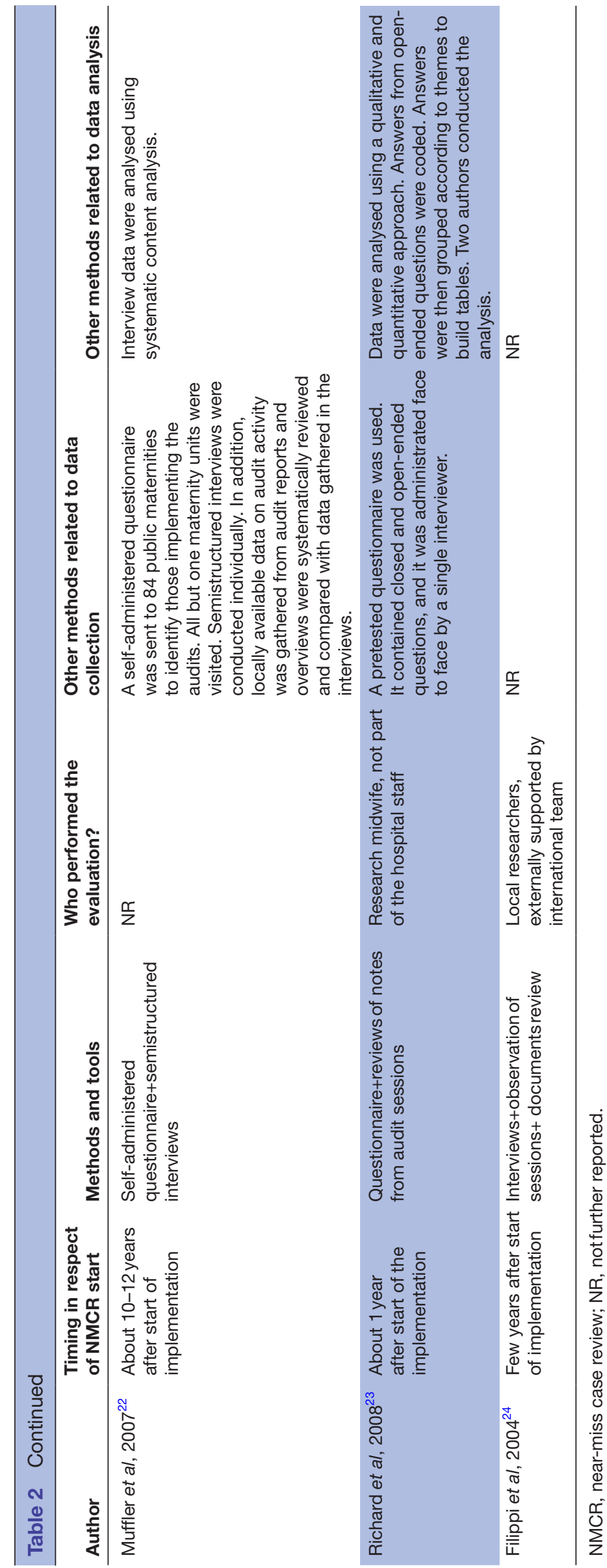

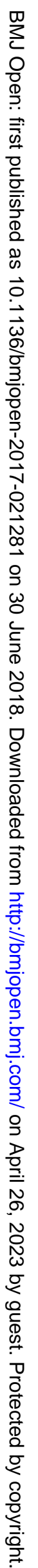




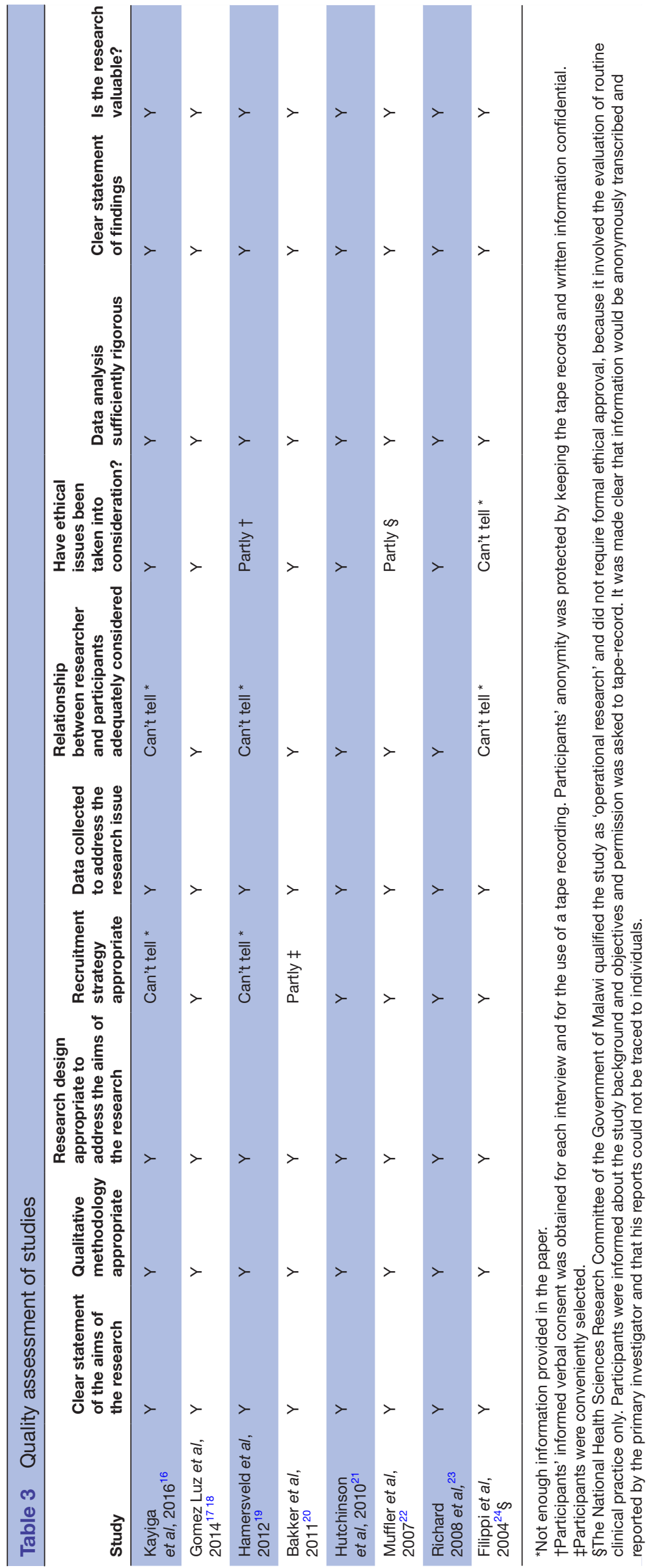

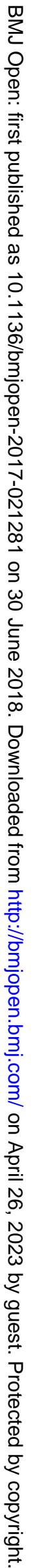




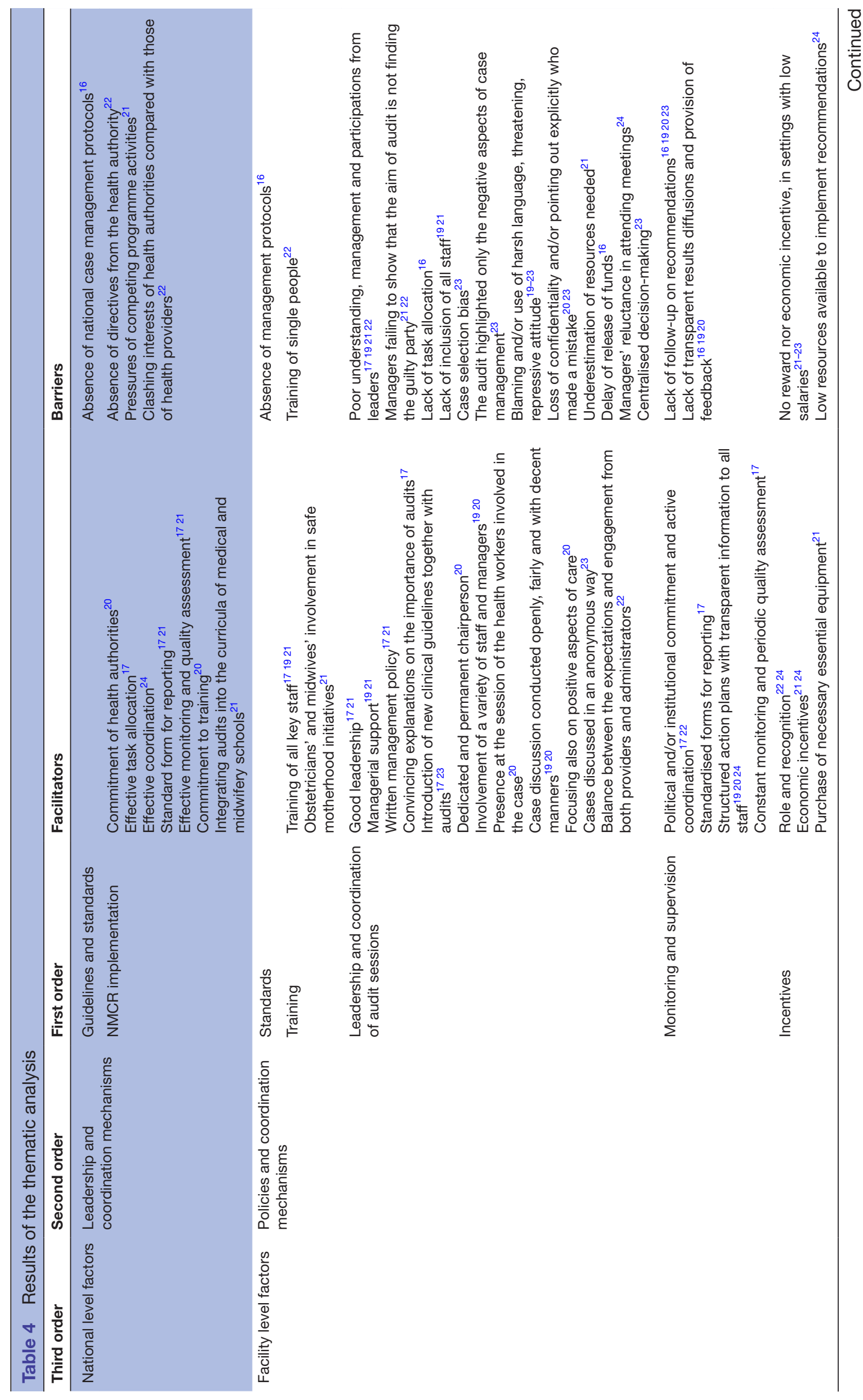




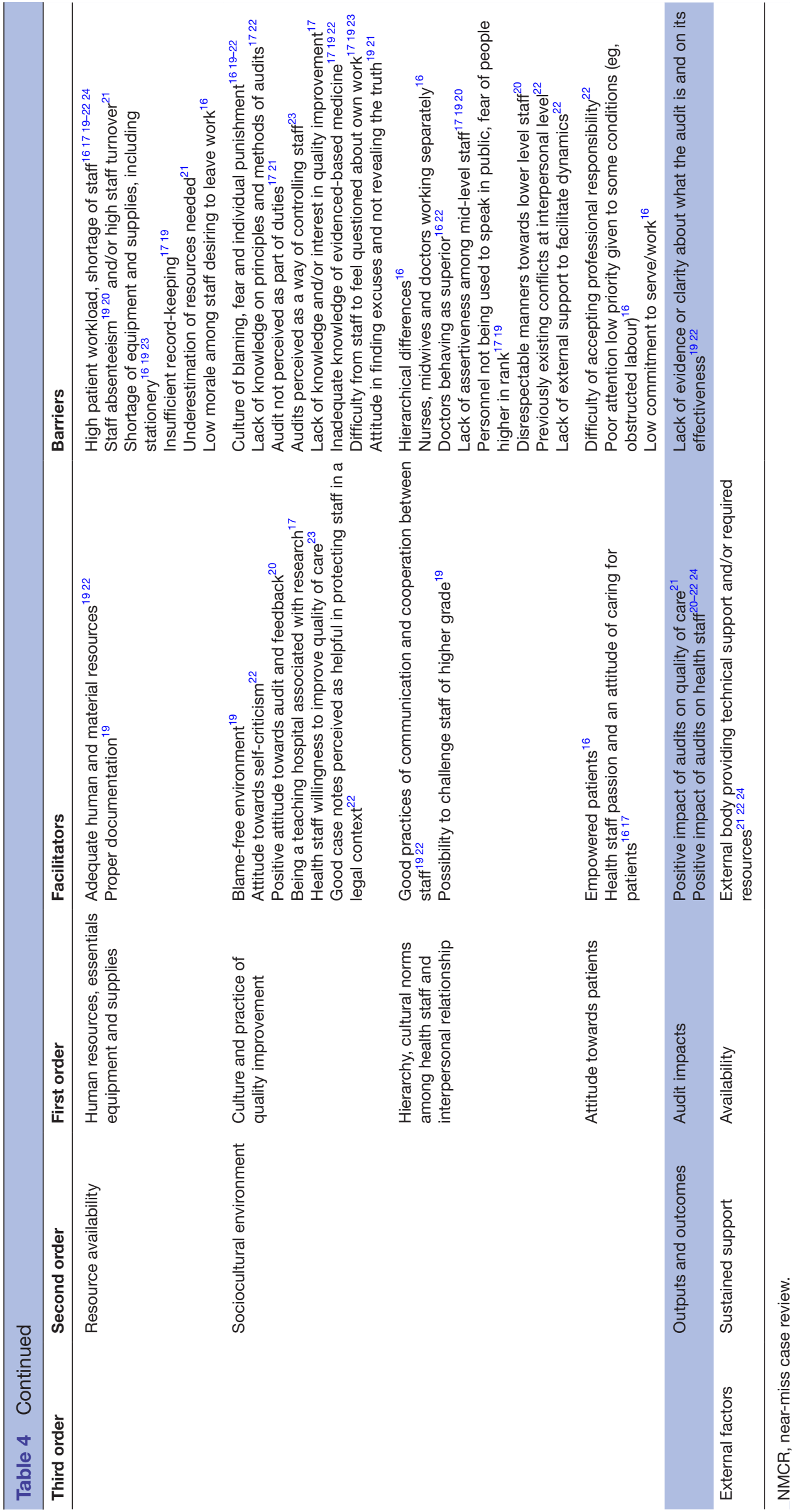

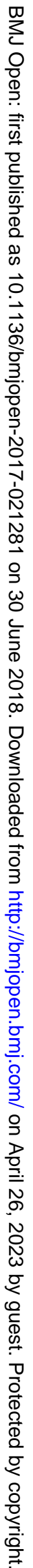




\section{Training}

Training of all key staff and managers on the principles, importance and methodology of the $\mathrm{NMCR}^{171921}$ was reported as key factor facilitating their implementation. In addition, programmes to strengthen involvement of obstetricians and midwives in safe motherhood initiatives $^{21}$ were reported as useful.

On the other side, however, training a limited number of people (most often, only the local coordinator/facilitator) meant there was a risk of the process to be entirely dependent on the availability of that single person ${ }^{22}$ and this was noted as a barrier.

\section{Leadership and coordination of audit sessions}

A list of factors related to leadership and coordination was reported as facilitators to case reviews: good leadership $^{1721}$; managerial support ${ }^{1921}$; existence of a written management policy ${ }^{17}$; ; clear and convincing explanation on the importance of audits ${ }^{17}$; leadership for the introduction of new clinical guidelines as opposed to audits only ${ }^{17} 23$; availability of a dedicated and permanent chairperson ${ }^{20}$; involvement of a variety of staff and managers in all stages of audit, with unrestricted admission to sessions ${ }^{1920}$; attendance to the session of the health workers who had been involved in the case management ${ }^{20}$; case discussion conducted openly and fairly with participants maintaining respect and good manners towards each other ${ }^{19}{ }^{20}$; focus also on positive aspects of care $^{20}$; case discussion conducted in an anonymous way ${ }^{23}$ and finally a balance between the expectations and engagement from both providers and administrators. ${ }^{22}$

Similarly, a list of barriers related to leadership and coordination was reported, such as poor understanding from leaders of the NMCR process; poor leadership and lack of involvement of directors ${ }^{17}{ }^{19}{ }^{22}$; failure from managers in recognising that the NMCR aim is not finding who is guilty, but rather improving services ${ }^{21} 22$; lack of task allocation ${ }^{16}$; lack of inclusion of all types of staff (eg, midwives, laboratory services) and poor participation of certain type of staff (eg, doctors or low-level staff not attending or attending irregularly) ${ }^{1921}$; case selection bias (eg, selecting only cases where mid-level staff, but not doctors, committed mistakes) ${ }^{23}$; highlighting only the negative aspects of case management ${ }^{23}$; blaming and/or using harsh language or bossing attitude ${ }^{19-23}$; loss of confidentiality during the sessions ${ }^{23}$; managers reluctance to attend meetings for fear of requests they cannot fulfil. ${ }^{24}$ Other barriers included delay in releasing funds ${ }^{16}$ and centralised human resources management and decision-making inhibiting initiatives by the clinicians. ${ }^{23}$

\section{Monitoring and supervision}

Political and/or institutional commitment in monitoring and supervision, active coordination of accountability mechanisms, ${ }^{1722}$ together with the availability of standardised forms for reporting, ${ }^{17}$ structured action plans to implement the NMCR recommendations with transparent information to all staff members, ${ }^{19} 2024$ effective monitoring, periodic quality assessment and networking of local teams to a central coordinating centre $^{17}$ were reported by staff as facilitators of the NMCR implementation.

On the other side, lack of follow-up on recommendations ${ }^{16192023}$ and lack of transparent results dissemination and provision of feedback ${ }^{161920}$ were cited as barriers.

\section{Incentives}

Incentives such as appointing a role ${ }^{22} 24$ or providing some form of recognition such as economic incentives for participating in the audit sessions, ${ }^{21}{ }^{24}$ and purchasing necessary essential equipment as recommended from the case reviews ${ }^{21}$ were observed as important factors to allow NMCR sustainability over time.

On the contrary, the absence of a reward or of an economic incentive, even if minimal, in setting with low salaries and high inflation, ${ }^{21-23}$ together with the low resources available to implement recommendations ${ }^{24}$ were perceived as key barriers.

\section{Resource availability}

Adequate human and material resources ${ }^{19} 22$ and proper documentation ${ }^{19}$ were reported as essentials to carry forward the NMCR.

On the other side, high patients workload, shortage of staff, ${ }^{161719-22} 24$ staff absenteeism ${ }^{1920}$ and/or high staff turnover, ${ }^{21}$ together with shortage of equipment and supplies, including stationery, ${ }^{16}{ }^{19} 23$ insufficient recordkeeping $^{1719}$ and underestimation of resources needed ${ }^{21}$ were all perceived as barriers, associated with low morale among staff and desire to leave work. ${ }^{16}$

\section{Culture and practice of quality improvement}

A long list of sociocultural factors was reported as being either a facilitator or a barrier to effective implementation of NMCR. Factors perceived as facilitators were the following: a blame-free environment ${ }^{19}$; a culture of self-reflection among health workers and a general positive attitude towards audit and feedback ${ }^{20}{ }^{22}$; being a teaching hospital associated with research, ${ }^{17}$ motivational factors such as a desire to improve quality among healthcare personnel. ${ }^{23}$ Finally, staff's understanding that good quality in case management and appropriate documentation can help protect them in the case of a legal litigation $^{22}$ was also reported as a facilitator.

The list of sociocultural barriers included: a culture of blaming, fear and individual punishment ${ }^{16}{ }^{19-22}$; lack of knowledge on the principles and methods of audits ${ }^{17}$;2; the fact that NMCRs were not perceived as being part of regular duties ${ }^{1721}$ or that they were perceived as a way of controlling staff ${ }^{23}$; lack of knowledge and/or interest in quality improvement ${ }^{17}$; and inadequate knowledge on principles, methods and contents of evidence-based medicine. ${ }^{17} 1922$ These factors were reported as being associated with difficulties from staff when questioned about their own work, ${ }^{171923}$ and an attitude of making up 
excuses and not withholding the truth about what actually happened during the care of near-miss cases. ${ }^{19} 21$

\section{Hierarchy, cultural norms among health staff and interpersonal relationships}

Good practices of communication and cooperation between different cadres of health workers ${ }^{19} 22$ and the possibility of challenging a higher-level staff $^{19}$ were reported as facilitators of the NMCR implementation.

On the other side, barriers were perceived as following: the existence of hierarchical differences ${ }^{16}$; nurses, midwives and doctors working separately as opposed to acting as part of a team ${ }^{16}$; doctors' feeling/behaving as superior compared with other levels of staffing ${ }^{162}$; disrespectful manners towards lower-level staff ${ }^{20}$; lack of assertiveness among mid-level staff ${ }^{171920}$; staff not being used to speak in public, fear of talking in presence of staff in a higher rank ${ }^{17}{ }^{19}$; previously existing conflicts at interpersonal level ${ }^{22}$ as well as lack of external support to facilitate these dynamics. ${ }^{22}$

\section{Attitude towards patients and medical conditions}

The existence of a sufficient degree of empowerment among patients, patients having a recognised status and being respected, ${ }^{16}$ together with a caring attitude from the $\operatorname{staff}^{1617}$ were reported as facilitators of the NMCR implementation.

On the other side, difficulty of accepting professional responsibility, ${ }^{22}$ poor attention and low priority given to some clinical conditions possibly leading to complications (eg, obstructed labour) ${ }^{16}$ together with a low commitment to serve/work ${ }^{16}$ were reported as barriers.

\section{Outputs and outcomes}

Several studies reported that sustainability of audits also depended on their perceived effects. Where healthcare staff perceived that audits had a positive impact on quality of care-such as maternal or perinatal outcomes, respect for women's rights during childbirth, availability of equipment and organisation of care- ${ }^{21}$ and/or a positive impact on healthcare staff dynamics—such as improved communication and coordination, improved acceptance of responsibilities, increased awareness of problems, improved knowledge and skills ${ }^{20-22} 24$ - these factors facilitated the NMCR implementation over time.

On the other side, a lack of evidence or clarity about what the NMCR was, and on its effectiveness ${ }^{19} 22$ was perceived as a barrier to sustain the case reviews.

External partners factors

Sustained support

The existence of an external body or organisation able to provide technical support, and if needed additional required resources ${ }^{21} 2224$ were reported as a key factor to ensure effective NMCR implementation in different settings.

\section{Key recommendations}

Table 5 synthesises key recommendations for effective NMCR implementation. Actions are divided in those that may be implemented in the short term and those needing a longer time for the implementation but that may result in a longer-term impact.

\section{DISCUSSION}

This review fills a gap in evidence synthesis on facilitators and barriers to effective implementation of NMCR. Findings of the review suggest that the effective implementation of NMCR in maternity hospitals is a complex intervention that can be challenged by a number of barriers at different levels (national, facility, external partner level), including technical aspects (such as leadership and coordination mechanisms), resource availability (adequate human resources to manage workload and essential supplies), sociocultural factors (such as existing cultural norms, hierarchy among healthcare staff and patients' empowerment) and the lack of external support. On the other side, a number of facilitating factors were identified. Findings from this systematic review suggest a list of practical recommendations (table 5), which can be used by policy-makers and managers to prevent and mitigate common challenges to successful NMCR implementation.

This review was conducted according to the PRISMA ${ }^{8}$ and the ENTREQ ${ }^{9}$ standards. A broad search strategy in a large number of electronic databases was used. The key limitation of the review is the paucity of existing relevant scientific reports: although the NMCR approach has been used in many countries, there has been relatively few formal studies exploring facilitators and barriers to effective NMCR implementation. Despite the above-described limitation, this review retrieved an appreciable number of good-quality studies from the African Region. Findings of the review are therefore mostly generalisable to this setting.

Outside the African Region, we retrieved several informal evaluations reporting on enablers and barriers to effective NMCR implementation in Europe, Central Asia, South East Asia, Latin America and the Caribbean ${ }^{25-37}$ It will be inappropriate to pull together results of peer-reviewed formal studies with those of unpublished technical reports and informal evaluations. However, it may be interesting to acknowledge that grey literature ${ }^{25-37}$ suggests that key factors enabling effective NMCR implementation in countries other than the African Region are similar to those observed in this review, with some peculiarities specific to each context. First, the importance of good leadership is a recurrent theme highlighted virtually in all grey literature. ${ }^{25-37}$ Second, the crucial role of a positive cultural environment has been reported as a key determinant of successful NMCR implementation on a global scale. ${ }^{25-36}$ For example, a review of experiences of NMCR implementation supported by the International Federation for Gynecology and Obstetrics in Europe, Asia and Africa identified three independent cultural factors as key determinants for the successful NMCR implementation: (1) individual responsibility and ownership; (2) a proactive institutional ethos, promoting learning as a 
Table 5 Key recommendations for effective NMCR implementation

Short term Long term

\section{External partners}

Ensure technical support.

\section{National level}

- Ensure general commitment and understanding of national and local health authorities.

- Ensure financial resources.

- Make available updated evidenced-based national guidelines and standards.

- Develop a good action plan and budget, covering all WHO recommendations. *

- Create the legal framework.

- Ensure effective leadership and coordination.

- Ensure timely monitoring and evaluation.

- Support timely transparent results dissemination to health staff and the community.

- Promote local responsibility and ownership.

- Collaborate with an external body for quality assessment.
Local level
- Ensure commitment, understanding and active participation of hospital directors.
- Dissemination of updated evidenced-based national guidelines and standards.
Develop a good action plan and budget, covering all WHO recommendations, ${ }^{5}$ considering feasibility based on local resources.
- Inform and create awareness among all staff.
- Train and adequate number and type of staff.
- Consider ways to provide some form of professional recognition for health staff involved in NMCR.
- Ensure effective leadership and coordination.
- Ensure that NMCR sessions are carried forward according the WHO recommendations. ${ }^{5}$
- Ensure that recommendations from the NMCR are put in place.
Ensure timely transparent results dissemination to all staff.

*See the WHO manual: WHO. Regional Office for Europe. Conducting a maternal near-miss case review cycle at the hospital level' manual with practical tools. Available at http://www.euro.who.int/en/health-topics/Life-stages/maternal-and-newborn-health/publications/2016/ conducting-a-maternal-near-miss-case-review-cycle-at-hospital-level-2016 NMCR, near-miss case review.

crucial part of improving services and (3) a supportive political and policy environment at both national and local levels. ${ }^{25}$ On the other side, identified cultural barriers for performing NMCR included a culture of blaming, fear and individual punishment, together with a lack of professionalism. ${ }^{25}$ Similarly, reports on NMCR implementation in ex-Soviet countries identified a culture of blaming, fear and individual punishment, and hierarchy among staff as key barriers for successful NMCR implementation. ${ }^{28-32}$ In ex-Soviet countries, the key element in promoting a safe, friendly, confidential environment was the emanation from Ministry of Health of prikazes (national laws) and the commitment of hospital directors to a non-punitive system. $^{3536}$

In line with what has been observed in this review, grey literature reporting experiences of NMCR implementation in LMIC in Europe and Asia deemed as crucial to provide some professional recognition for health staff involved in the case reviews. ${ }^{25} 2733$ In settings with very low resources, a small financial incentive was reported as essential, since in these contexts any non-paid activity outside working hours means a serious loss of income. ${ }^{21}$
Again, similarly to what has been reported in studies included in this review, ${ }^{19}$ the importance for staff to perceive clearly the potential and/or actual benefits of the audits (eg, improvements in quality of care, organisation of care, staff knowledge and recognition) was recognised as a key determinant of successful NMCR implementation in a number of reports from different regions, ${ }^{37}$ while disillusion from lack of actions following the reviews was highlighted as a important barrier for NMCR sustainability. ${ }^{25-28}$

Lack of knowledge of the evidence-based maternal and perinatal practices was reported as a barrier to NMCR implementation in the WHO European region, ${ }^{29}$ as well in studies in this review. As far as different types of hospitals were concerned, reports from both Europe, Latin America and Africa observed that the implementation of NMCR was easier in lower level facilities ${ }^{162433}$ or research hospitals ${ }^{17}$ where staff was used to work together, rather than in large maternity units dominated by 'academic tradition' difficult to challenge ${ }^{33}$ or where there was high staff turnover. ${ }^{16}$ Poor patient empowerment and insufficient inclusion of service user views were reported as 
barriers to successful NMCR implementation in Europe, Asia and Africa. ${ }^{252733}$ Finally, the availability of an external partner/organisation capable of providing sustained technical support (and, if needed, the resources to put in place the quality improvement recommendations) was a key factor mentioned in many reports from different countries. ${ }^{25} 27-30323536$

This review contributes to the current debate on quality improvement interventions and on the knowledge of potential challenges to their implementation. When compared with other systematic reviews of facilitators and barriers of effective implementation of other quality improvement interventions, ${ }^{38} 39$ it appears that, not surprising, many barriers, such as the lack of coordination and leadership or lack of knowledge of evidence-based practices, are common to different quality improvement interventions. More research should be conducted to test strategies aiming at facilitating successful implementation for NMCR as well as for other quality improvement interventions.

\section{CONCLUSIONS}

Studies suggest that the effective implementation of NMCR at facility level is a complex intervention that can be challenged by a number of barriers at different levels (national, facility level, external partner level). Policy-makers, in planning the NMCR implementation, should consider the lessons learnt from previous studies as synthesised in this paper and should carefully plan actions to prevent and mitigate common challenges to successful NMCR implementation. Future studies should aim at documenting better facilitators and barriers to successful implementation of the facility-based individual NMCR, especially outside the African region, as well as exploring facilitators and barriers for other quality improvement interventions, and in testing strategies aiming at facilitating successful implementation.

Acknowledgements We thank Sonia Richardson for having reviewed the English language of this manuscript.

Contributors ML conceived the papers, extracted data, analysed data, drafted the first version of this paper and finalised the final version. SR screened the studies and revised the first draft. BC and MC extracted data, analysed data and revised the first draft.

Funding This review was funded by a grant from the GREAT Network, Canadian Institutes of Health Research, St. Michael's Hospital, Toronto.

Competing interests None declared.

Patient consent Not required.

Provenance and peer review Not commissioned; externally peer reviewed.

Data sharing statement All key data are provided in the paper. Additional details can be provided by the contact author on request.

Open access This is an open access article distributed in accordance with the Creative Commons Attribution Non Commercial (CC BY-NC 4.0) license, which permits others to distribute, remix, adapt, build upon this work non-commercially, and license their derivative works on different terms, provided the original work is properly cited and the use is non-commercial. See: http://creativecommons.org/ licenses/by-nc/4.0/ (c) Article author(s) (or their employer(s) unless otherwise stated in the text of the article) 2018. All rights reserved. No commercial use is permitted unless otherwise expressly granted.

\section{REFERENCES}

1. World Health Organization. Global Strategy for Women's, Children's and Adolescent's Health 2016-2030. http://www.who.int/life-course/ partners/global-strategy/global-strategy-2016-2030/en/ (accessed 15 Sep 2016).

2. Tunçalp Ö, Were WM, MacLennan C, et al. Quality of care for pregnant women and newborns-the WHO vision. BJOG 2015;122:1045-9.

3. World Health Organization (WHO). The prevention and elimination of disrespect and abuse during facility-based childbirth. Geneva: World Health Organization, 2014

4. World Health Organization. Beyond the numbers: Reviewing maternal deaths and complications to make pregnancy safer. Geneva: World Health Organization, 2004

5. World Health Organization. Regional Office for Europe. Conducting a maternal near-miss case review cycle at the hospital level" manual with practical tools. http://www.euro.who.int/en/health-topics/Lifestages/maternal-and-newborn-health/publications/2016/conductinga-maternal-near-miss-case-review-cycle-at-hospital-level-2016 (accessed 29 Nov 2016)

6. Tunçalp O, Hindin MJ, Souza JP, et al. The prevalence of maternal near miss: a systematic review. BJOG 2012;119:653-61.

7. Lazzerini M, Richardson S, Ciardelli V, et al. Effectiveness of the facility-based maternal near-miss case reviews in improving maternal and newborn quality of care in low-income and middle-income countries: a systematic review. BMJ Open 2018;8:e019787.

8. Liberati A, Altman DG, Tetzlaff J, et al. The PRISMA statement for reporting systematic reviews and meta-analyses of studies that evaluate health care interventions: explanation and elaboration. PLOS Med 2009;6:e1000100.

9. Tong A, Flemming K, Mclnnes E, et al. Enhancing transparency in reporting the synthesis of qualitative research: ENTREQ. BMC Med Res Methodol 2012;12:181.

10. The World Bank. Country and Lending Groups. Historical classification. $2014 \mathrm{https} / / /$ datahelpdesk.worldbank.org/ knowledgebase/articles/906519 (accessed 2 Nov 2017).

11. World Health Organization. Evaluating the quality of care for severe pregnancy complications: the WHO near-miss approach for maternal health. Geneva: World Health Organization, 2011.

12. Thomas J, Harden A. Methods for the thematic synthesis of qualitative research in systematic reviews. BMC Med Res Methodol 2008;8:45.

13. Bohren MA, Hunter EC, Munthe-Kaas HM, et al. Facilitators and barriers to facility-based delivery in low- and middle-income countries: a qualitative evidence synthesis. Reprod Health 2014;11:71

14. Glenton C, Colvin CJ, Carlsen B, et al. Barriers and facilitators to the implementation of lay health worker programmes to improve access to maternal and child health: qualitative evidence synthesis. Cochrane Database Syst Rev 2013;10:CD010414.

15. Critical Appraisal Skills Programme (CASP). Quality-assessment tool for qualitative studies. http://www.casp-uk.net/\#!casp-toolschecklists/c18f8 (accessed on 26 Jan 2015).

16. Kayiga $\mathrm{H}$, Ajeani J, Kiondo $\mathrm{P}$, et al. Improving the quality of obstetric care for women with obstructed labour in the national referral hospital in Uganda: lessons learnt from criteria based audit. BMC Pregnancy Childbirth 2016;16:152.

17. Luz AG, Osis MJ, Ribeiro M, et al. Impact of a nationwide study for surveillance of maternal near-miss on the quality of care provided by participating centers: a quantitative and qualitative approach. $B M C$ Pregnancy Childbirth 2014;14:122.

18. Luz AG, Osis MJ, Ribeiro M, et al. Perspectives of professionals participating in the Brazilian Network for the Surveillance of Severe Maternal Morbidity regarding the implementation of routine surveillance: a qualitative study. Reprod Health 2014;11:29.

19. van Hamersveld KT, den Bakker E, Nyamtema AS, et al. Barriers to conducting effective obstetric audit in Ifakara: a qualitative assessment in an under-resourced setting in Tanzania. Trop Med Int Health 2012;17:652-7.

20. Bakker W, van den Akker T, Mwagomba B, et al. Health workers' perceptions of obstetric critical incident audit in Thyolo District, Malawi. Trop Med Int Health 2011;16:1243-50. 
21. Hutchinson C, Lange I, Kanhonou L, et al. Exploring the sustainability of obstetric near-miss case reviews: a qualitative study in the South of Benin. Midwifery 2010;26:537-43.

22. Muffler N, Trabelssi MH, De Brouwere V. Scaling up clinical audits of obstetric cases in Morocco. Trop Med Int Health 2007;12:1248-57.

23. Richard $F$, Ouédraogo $C$, Zongo $V$, et al. The difficulty of questioning clinical practice: experience of facility-based case reviews in Ouagadougou, Burkina Faso. BJOG 2009;116:38-44.

24. Filippi V, Brugha R, Browne E, et al. Obstetric audit in resource-poor settings: lessons from a multi-country project auditing 'near miss' obstetrical emergencies. Health Policy Plan 2004;19:57-66.

25. Lewis $\mathrm{G}$. The cultural environment behind successful maternal death and morbidity reviews. BJOG 2014;121(Suppl 4):24-31.

26. Lewis G. Emerging lessons from the FIGO LOGIC initiative on maternal death and near-miss reviews. Int J Gynaecol Obstet 2014;127(Suppl 1):S17-20.

27. Bacci A, Lewis G, Baltag V, et al. The introduction of confidential enquiries into maternal deaths and near-miss case reviews in the WHO European Region. Reprod Health Matters 2007;15:145-52.

28. Bacci A, Hodorogea S, Khachatryan $\mathrm{H}$, et al. What is the quality of the maternal near-miss case reviews in WHO European Region? Cross-sectional study in Armenia, Georgia, Latvia, Republic of Moldova and Uzbekistan. BMJ Open 2018;8:e017696.

29. World Health Organization. Regional Office for Europe. Multi-Country review meeting on maternal mortality and morbidity audit "Beyond the Numbers", Report of a WHO meeting, Charvak, Uzbekistan 14-17 June 2010. Copenhagen: WHO Regional Office for Europe, 2010.

30. World Health Organization. Regional Office for Europe. The impact of implementation of 'Beyond the numbers' approach in improving maternal and perinatal health. Bishkek, Kyrgyzstan. Copenhagen: WHO Regional Office for Europe, 2014.
31. WHO Regional Office for Europe Making Pregnancy Safer in Uzbekistan. Maternal mortality and morbidity audit Activities Report 2002-2008. http://www.euro.who.int/_data/assets/pdf_file/0004/ 98797/MPS UZB.pdf (accessed 8 Nov 2016).

32. Sukhanberdiyev K, Ayazbekov A, Issina A, et al. Initial experience of Near Miss Case Review: improving the management of haemorrhage. Entre Nous 2011;74:18-19.

33. Baltag V, Filippi V, Bacci A. Putting theory into practice: the introduction of obstetric near-miss case reviews in the Republic of Moldova. Int J Qual Health Care 2012;24:182-8.

34. Borchert M, Goufodji S, Alihonou E, et al. Can hospital audit teams identify case management problems, analyse their causes, identify and implement improvements? A cross-sectional process evaluation of obstetric near-miss case reviews in Benin. BMC Pregnancy Childbirth 2012;12:109.

35. Hodorogea S. Piloting near miss case reviews in Kazakhstan: improving quality of maternal care. Entre Nous 2010;70:28-9.

36. Yadgarova K, Fazilova F. Implementation of near miss case reviews in Uzbekistan: the role of prikazes. Entre Nous 2010;70:26-7.

37. Bailey PE, Binh HT, Bang HT. Promoting accountability in obstetric care: use of criteria-based audit in Viet Nam. Glob Public Health 2010;5:62-74.

38. Stokes T, Shaw EJ, Camosso-Stefinovic J, et al. Barriers and enablers to guideline implementation strategies to improve obstetric care practice in low- and middle-income countries: a systematic review of qualitative evidence. Implement Sci 2016;11:144.

39. Allanson ER, Tunçalp Ö, Vogel JP, et al. Implementation of effective practices in health facilities: a systematic review of cluster randomised trials. BMJ Glob Health 2017;2:e000266 